\title{
The use of COLD-PCR and pyrosequencing for sensitive detection of EGFR T790M mutation
}

\author{
Huimin Chen ${ }^{1}$, Yang Zhang ${ }^{1}$, Dan $\mathrm{Pu}^{1, *}$, and Kunxian Shu ${ }^{1}$ \\ ${ }^{1}$ Chongqing Key Laboratory of Big Data for Bio Intelligence, Chongqing University of Posts and Telecommunications, Chongqing, \\ China
}

\begin{abstract}
A sensitive and convenient method for the detection of epidermal growth factor receptor (EGFR) T790M mutation in non-small cell lung cancer (NSCLC) patients with acquired resistance to tyrosine kinase inhibitors (TKIs) would be desirable to guide treatment strategy. Consequently, studies have focused on sensitive characterization of EGFR T790M mutation. Herein, two methods of co-amplification at lower denaturation temperature PCR (COLD-PCR) and pyrosequencing were combined (COLDPCR/pyrosequencing) for detecting EGFR T790M mutation. Evaluation of mutation-containing dilutions revealed that the sensitivities of COLD-PCR/pyrosequencing and conventional PCR/pyrosequencing assays for the detection of the T790M mutation were 0.1 and $5 \%$, respectively, indicating a 50 -fold increase in sensitivity. When the T790M mutation in 20 clinical NSCLC samples who had relapsed under firstgeneration EGFR TKI were further determined using COLD-PCR/pyrosequencing and conventional $\mathrm{PCR} /$ pyrosequencing, the detection rates were $35 \%(7 / 20)$ and $25 \%(5 / 20)$, respectively. All patients who were positive for the T790M mutation with conventional $\mathrm{PCR} /$ pyrosequencing were also found to be positive with COLD-PCR/pyrosequencing. The discordant cases were 2 samples with no T790M mutation detected with conventional PCR/pyrosequencing, but which were positive with COLD-PCR/pyrosequencing. COLD-PCR/pyrosequencing is a sensitive and cost-effective tool for detecting the T790M mutation which will permit an improvement of therapeutic management.
\end{abstract}

\section{Introduction}

In recent years, the use of epidermal growth factor receptor tyrosine kinase inhibitors (EGFR-TKIs) has significantly improved the survival of patients with nonsmall cell lung cancer (NSCLC) harboring activating EGFR mutations ([1]). However, most patients eventually acquire resistance to the drug, resulting in disease progression ([2]). Half of all resistance to EGFRTKIs is caused by a threonine-methionine amino acid substitution at position 790 (T790M) in exon 20 of $E G F R$ gene ([3]). Thus, it is clinically crucial to detect T790M mutation in exon 20 of the EGFR gene. However, the ability to detect $E G F R$ T790M mutation can be difficult since the specimen samples retrieved from tumor tissues and liquid biopsies such as cell-free DNA (cfDNA) may be low in tumor abundance or DNA concentration. As a result, accurate and sensitive molecular techniques and methods for EGFR T790M mutation analysis are acquired. The current methods for detecting of T790M mutation in clinical practice include real-time PCR assays ([4]), allele-specific (AS) PCR ([5]), amplification refractory mutation system (ARMS) ([1]), droplet digital PCR (ddPCR) ([1, 5]), highresolution melting (HRM) ([6]), Sanger sequencing ([6]) and next-generation sequencing (NGS) ([7]). Among those PCR-based methods, some do not allow to detect low-abundance $E G F R$ T790M mutation, while others are highly sensitive. But there is a potential for false-positive results. These false-positive results might result from the amplifications of small amounts of DNA ([8,9]). In addition, HRM is a simple, rapid and sensitive method for detecting EGFR T790M mutation, however it is hampered by the inability to identify the specific nucleotide change ([10]). Sanger sequencing enables to interrogate the specific nucleotide change, whereas it has a low analytical sensitivity of $20 \%$ of the mutated allele in a WT background ([11]). NGS is another sequencing technique enabled to mutation detection, but it is high cost, time-consuming and requiring a large amount of sequencing to detect rare mutations, rendering it not suitable for clinical routine use in a lot of laboratories ([12]). Therefore, finding a sensitive and convenient detection assay is important.

Pyrosequencing also has the potential for mutation detection. It is a sequencing-by-synthesis (SBS) based method, in which one of the four nucleotides (A, G, C, and $\mathrm{T}$ ) is sequentially incorporated at a time during a cyclic primer extension step. Each allelic variant will give a unique peak compared to the two other variants, which allows to specifically discriminate different alleles by use of pyrosequencing ([13]). Compared to Sanger sequencing, pyrosequencing is more sensitive with the limit of detection as low as $5 \%$ of the mutant allele in

\footnotetext{
* Corresponding author: pudan@cqupt.edu.cn
} 
wild-type DNA ([11]). Although having higher sensitivity in variant detection, pyrosequencing is unable to selectively enrich the variant alleles, which would be requested in detecting low percentage of mutated DNA.

Co-amplification at lower denaturation temperature PCR (COLD-PCR) is a modified PCR method which allows to selectively amplify low-abundance alleles from a mixture of wild-type and mutant sequences, irrespective of the mutation type and position ([14, 15]). It reduces the limitations in low-abundance mutation detection by use of a critical denaturation temperature $\left(T_{\mathrm{c}}\right)$ during PCR amplification. Low-abundance mutation enrichment by use of COLD-PCR has been demonstrated in combination with several downstream approaches such as TaqMan-based real-time PCR ([16]), HRM ([17]), Sanger sequencing ([6]) and pyrosequencing ([14]). When combined with pyrosequencing, COLD-PCR can improve mutation detection and identification by approximately 5-fold to 35-fold ([14]).

Here, we present the combination of COLD-PCR with pyrosequencing for the detection of EGFR T790M mutation. We demonstrate that upstream COLD-PCR assay allows for the enrichment of EGFR T790M mutation in a sensitive and inexpensive manner and downstream pyrosequencing of COLD-CPR products allows for the discrete identification of low-abundance EGFR T790M mutation.

\section{Materials and methods}

\subsection{Samples and DNA extraction}

Tumor specimens were obtained from 20 NSCLC patients from Southwest Hospital who had relapsed under first-generation EGFR TKI. For all the specimens, the T790M mutation in EGFR gene was assessed by $\mathrm{PCR} /$ pyrosequencing and 5 results were positive. Genomic DNA from formalin-fixed paraffin-embedded (FFPE) tissue was isolated using QIAamp DNA FFPE Tissue Kit (Qiagen) following the manufacturer's instructions. After extraction and purification, DNA was quantified by a Qubit fluorometer (Thermo Fisher, Waltham, MA).

\subsection{Plasmid construction}

Plasmid standards were generated containing EGFR exon 20 with the T790M mutation. A plasmid containing wild-type EGFR exon 20 was also constructed. The sequences of the plasmids were confirmed by bidirectional Sanger sequencing. The constructed plasmids were then used to generate amplicons for positive and negative controls.

\subsection{Conventional PCR}

The templates harboring mutational sites were amplified using the following primers to obtain a 85-bp amplicon: Forward, 5'- CTGGGCATCTGCCTCACCT -3'; Reverse:
5'-biotin-TGTGTTCCCGGACATAGTCCA -3'. Primers were synthesized by Shenzhen Huada Gene Technology Co. LTD. (Shenzhen, China). Reactions for conventional PCR were performed using $25 \mathrm{ng}$ of genomic DNA in a total volume of $25-\mu \mathrm{L}$ containing a final concentration of $1 \times$ HotStar Taq DNA polymerase Buffer, $1.6 \mathrm{mM}$ of additional $\mathrm{MgCl}_{2}, 200 \mu \mathrm{M}$ of dNTPs, $200 \mathrm{nM}$ of each primer and $2 \mathrm{U}$ of HotStar TaqDNA polymerase. Amplification by conventional PCR was performed with an initial denaturing at $98{ }^{\circ} \mathrm{C}$ for $30 \mathrm{~s}, 40$ cycles of $98{ }^{\circ} \mathrm{C}$ for $10 \mathrm{~s}, 50.3{ }^{\circ} \mathrm{C}$ for $20 \mathrm{~s}$, and $72{ }^{\circ} \mathrm{C}$ for $10 \mathrm{~s}$, and final extension at $72{ }^{\circ} \mathrm{C}$ for $10 \mathrm{~min}$. The reactions are carried out on a $\mathrm{C} 1000$ Touch $^{\mathrm{TM}}$ Thermal Cycler (Bio-Rad, Hercules, CA, USA). After PCR amplification, electrophoresis on $2 \%$ agarose gel was performed to confirm successful amplification of the 85bp PCR product before pyrosequencing.

\subsection{Determination of critical denaturation temperature, $T_{\mathrm{c}}$}

The most important thing in designing a COLD-PCR assay is to determine the best $T_{\mathrm{c}}$ for the reaction. To define optimal $T_{\mathrm{c}}$ for the given amplicon with EGFR T790M mutation, several series of COLD-PCR assays with gradient $T_{\mathrm{c}}$ were performed to check different temperatures decreasing from 83.8 to $80.5^{\circ} \mathrm{C}$ using standard mixed plasmids at $3 \%$ mutation fraction. Direct sequencing of PCR product was used to determine the degree to which the mutant allele was enriched. The denaturation temperature that reproducibly produces good quality PCR amplicons with strong enrichment should be selected as the $T_{\mathrm{c}}$.

\subsection{COLD-PCR}

COLD-PCR can be performed in two formats, fullCOLD-PCR and fast-COLD-PCR ([14]). Here, fullCOLD-PCR was performed to enrich EGFR T790M mutation, since full-COLD-PCR enabled to identify all possible mutations. COLD-PCR was performed following the introduction in the literature ([14]), with minor modification. The COLD-PCR reaction mixtures consisted of $25 \mathrm{ng}$ of genomic DNA, $1 \times$ of Phusion DNA polymerase Buffer, $1.6 \mathrm{mM}$ of additional $\mathrm{MgCl}_{2}$, $200 \mu \mathrm{M}$ of dNTPs, $200 \mathrm{nM}$ of each primer and $5 \mathrm{U}$ of PhusionTM DNA polymerase (New England Biolabs (NEB)). The COLD-PCR protocol started with 5 cycles of the conventional PCR amplification for an initial buildup of all amplicons, followed by 39 COLD-PCR cycles to selectively enrich for mutant sequences. The full COLD-PCR cycling conditions included an initial denaturation for $30 \mathrm{~s}$ at $98{ }^{\circ} \mathrm{C}$, followed by 5 cycles of standard PCR $\left(10 \mathrm{~s}\right.$ denaturation at $98{ }^{\circ} \mathrm{C}, 20 \mathrm{~s}$ annealing at $50.3^{\circ} \mathrm{C}$, and $10 \mathrm{~s}$ elongation at $\left.72{ }^{\circ} \mathrm{C}\right)$ and 39 cycles of COLD-PCR ( $10 \mathrm{~s}$ denaturation at $98{ }^{\circ} \mathrm{C}, 30$ annealing at $70{ }^{\circ} \mathrm{C}, 10 \mathrm{~s}$ at $T_{\mathrm{c}}, 20 \mathrm{~s}$ primer annealing at $50.3^{\circ} \mathrm{C}$ and 10 s elongation at $72{ }^{\circ} \mathrm{C}$ ), followed by a final extension for 5 min at $72^{\circ} \mathrm{C}$. Amplicons were analyzed by gel electrophoresis on a agarose gel prior to pyrosequencing. 


\subsection{Pyrosequencing}

According to our previous work, $20 \mu \mathrm{L}$ of PCR or COLD-PCR products were purified and rendered singlestranded on a PyroMark Q96 Vacuum Workstation (Qiagen, Courtaboeuf, France) ([18]). Mutation detection was performed on a PyroMark Q96 MD (Qiagen, Hilden, Germany) following the manufacturer's instructions using the pyrosequencing primer, 5'CCGTGCAGCTCATCA-3'. Each sample was sequenced with nucleotide dispensation order: 5'GCTGCTAGC-3'. Quantitative mutation detection was analyzed with the Pyromark MS software (Qiagen) in the allele quantification (AQ) mode. After the run, Pyromark Q96 MA software was able to automatically analyze pyrogram (A series of peaks were called a pyrogram) outputs and the percentage of mutated amplicons was automatically shown upon the pyrogram. Each sample used for EGFR T790M analysis was also reanalyzed in triplicate to confirm the reproducibility.

\subsection{Determination of the sensitivity of COLD- $\mathrm{PCR} /$ pyrosequencing assays}

A plasmid DNA sample containing EGFR T790M mutation was used as the source of the mutant allele. This mutation-containing DNA sample was serially diluted into wild-type DNA to obtain the following percentages: $10,5, \quad 1,0.5,0.1,0.05$, and $0 \%$. Additionally, several replicates of wild-type DNA $(0 \%$ mutant) were included in each experiment and evaluated in parallel. All the DNA mixtures were simultaneously subjected to conventional PCR and COLD-PCR and then interrogated by pyrosequencing. All samples were run in duplicate.

\section{Results}

\subsection{Determination of optimal $T_{\mathrm{c}}$ for EGFR T790M detection}

When COLD-PCR is performed to enrich mutant sequence, the use of a very precise $T_{\mathrm{c}}$ is crucial, because a slight variation of as little as $0.2-0.3{ }^{\circ} \mathrm{C}$ from the $T_{\mathrm{c}}$ could make it difficult to obtain satisfactory mutation enrichment ([19]). Furthermore, for each DNA sequence, $T_{\mathrm{c}}$ is strongly dependent on DNA sequence, and it is determined practically for each specific sequence. To precisely determine the optimal $T_{\mathrm{c}}$ for EGFR T790M detection, COLD-PCR assay was evaluated using templates with $5 \%$ mutant-to-wild-type ratios and a range of lower denaturation temperatures $(80.5,81.4$, $81.9,82.7,83.3$, and $83.8^{\circ} \mathrm{C}$ ). Subsequently, amplification products were tested by pyrosequencing (Fig. 1). As shown, after COLD-PCR, 13, 14, 14, 15, 16, and $8 \%$ mutant-to-wild-type ratios were obtained at different $T_{\mathrm{c}}$. When lower denaturation temperature was
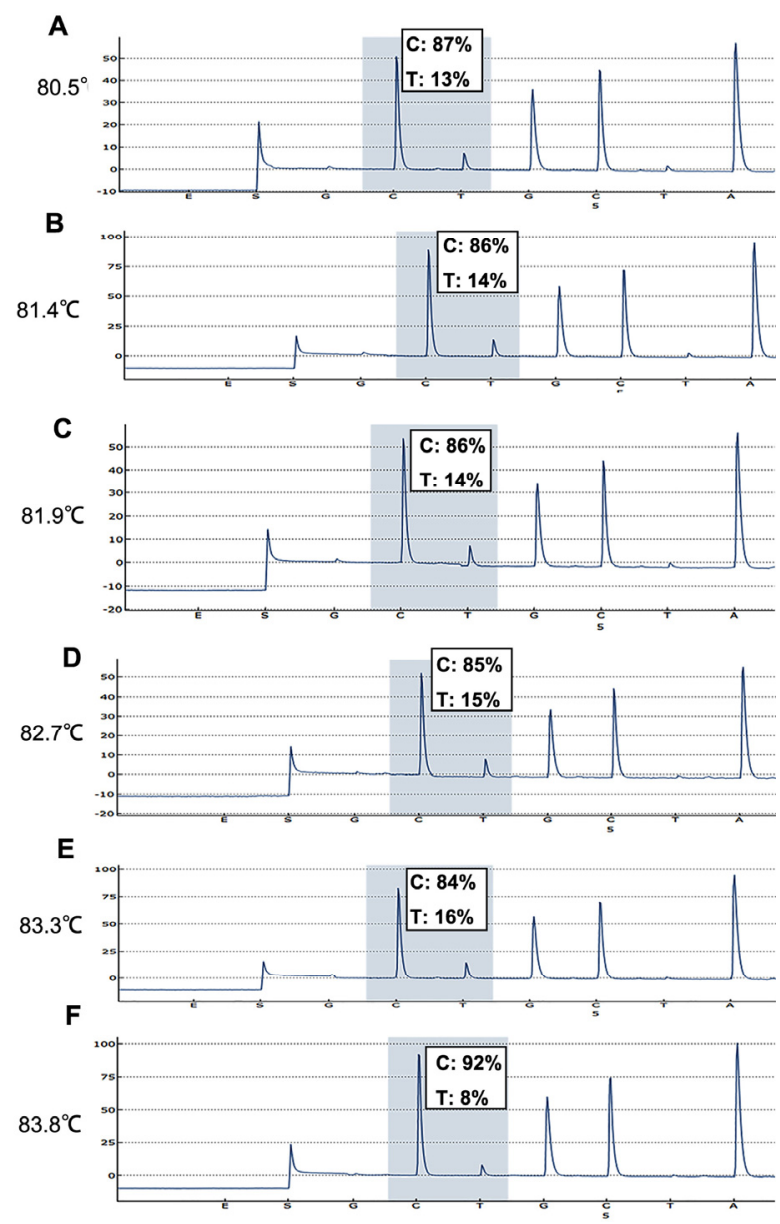

Fig. 1. The pyrograms from pyrosequencing after COLD-PCR. (A)-(F) lower denaturation temperatures applied was 80.5, 81.4, $81.9,82.7,83.3$, and $83.8^{\circ} \mathrm{C}$. As shown, $83.3^{\circ} \mathrm{C}$ was designed as the optimal $T_{\mathrm{c}}$.

set to $83.3^{\circ} \mathrm{C}$, the best mutant-sequence enrichment provided, with efficient amplification. Thus, the optimal $T_{\mathrm{c}}$ for $E G F R$ T790M detection was $83.3^{\circ} \mathrm{C}$.

\subsection{Sensitivity of COLD-PCR/pyrosequencing for EGFR T790M detection}

To evaluate the theoretical sensitivity of COLD$\mathrm{PCR} /$ pyrosequencing for detecting EGFR T790M mutation, a mutation-containing DNA sample was serially diluted with a wild-type DNA sample to obtain $10,5,1,0.5,0.1,0.05$ and $0 \%$ mutant-to-wild-type ratios. These reconstituted samples were simultaneously submitted to conventional PCR and COLD-PCR followed by pyrosequencing (Fig. 2). As shown, pyrosequencing analysis of COLD-PCR amplicons demonstrated more efficient enrichment of the T790M mutation. However, for conventional PCR, there is nearly no inherent potential for preferential enrichment. For example, in the pyrograms $10 \%$ initial EGFR T790M appeared to be enriched to $28 \%$ and $11 \%$ after COLD-PCR and conventional PCR, respectively. The pyrograms of amplicons obtained with conventional PCR demonstrated that the minimum detectable percentage was approximately $5 \%$, which was consistent 

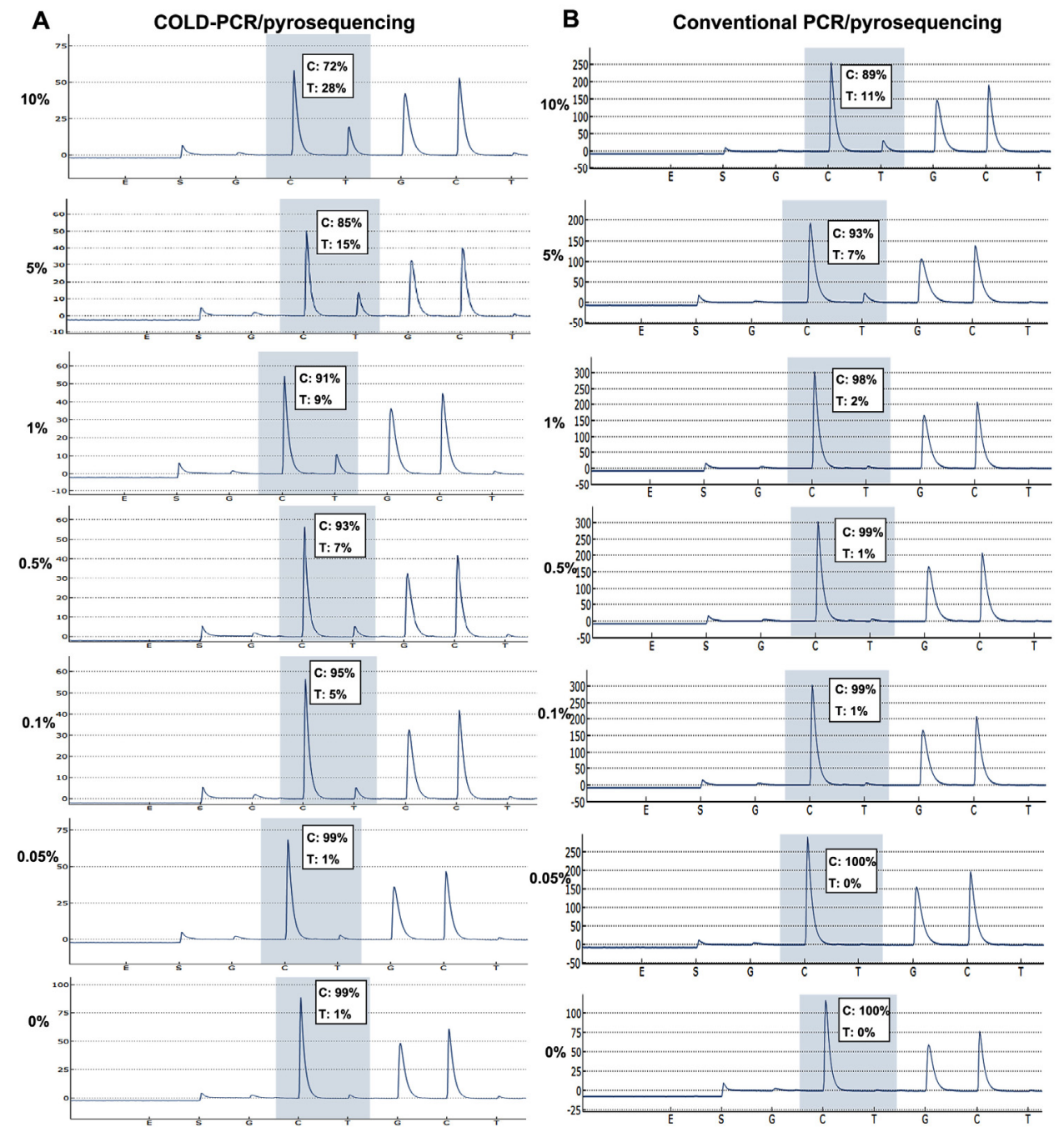

Fig. 2. Pyrosequencing analysis of the 85 -bp amplicon after COLD-PCR and conventional PCR, respectively. Pyrograms were presented for (A) COLD-PCR and (B) Conventional PCR. Serial dilutions (10, 5, 1, 0.5, 0.1, 0.05 and 0\%) of EGFR T790M mutation-containing DNA into wild-type DNA were presented.

with previous research results ([11]). Conversely, after COLD-PCR/pyrosequencing $0.1 \%$ EGFR T790M were easily detectable, providing a 50 -fold increase in the detection of this mutant.

We further evaluated the enrichment potential of COLD-PCR/pyrosequencing for EGFR T790M mutation detection. As shown in Fig. 3, when the initial abundance of EGFR T790M was larger than $0.5 \%$, lower abundance of initial mutation abundance exhibited a greater enrichment in COLD-PCR platforms, compared to the relatively higher mutant fractions. That was, within a certain abundance range $(>0.5 \%$ for $E G F R$ T790M mutation), the mutation enrichment of COLD$\mathrm{PCR} /$ pyrosequencing was highly related to the initial starting abundance of the EGFR T790M mutation.

\subsection{COLD-PCR/pyrosequencing for EGFR T790M detection in clinical samples}

We further examined the potential clinical applications of COLD-PCR/pyrosequencing over conventional $\mathrm{PCR} /$ pyrosequencing for detecting EGFR T790M mutation in clinical samples. 20 NSCLC patients who were first tested using conventional $\mathrm{PCR} /$ pyrosequencing to assess whether the T790M mutation was positive, and 5 results were positive. All the 20 NSCLC patients were further enriched by COLDPCR followed by pyrosequencing. As shown in Table 1, COLD-PCR/pyrosequencing successfully detected the T790M mutation in all the 5 samples that were positive by conventional PCR/pyrosequencing. Furthermore, among the 15 undetectable samples, 2 of them with no T790M mutation detected by conventional PCR/pyrosequencing were successfully detected by using COLD-PCR/pyrosequencing with the mutant-towild-type ratio of $10 \%$ and $8 \%$, respectively. That was, COLD-PCR/pyrosequencing increased the mutations detected in clinical NSCLC tumor specimens by $10 \%$ (2/20). Additionally, COLD-PCR/pyrosequencing enhanced the ratios of the mutant-to-wild-type approximately 1.4-2.6-fold. Thus, this protocol allowed for the detection of much lower abundant T790M mutations in comparison with conventional $\mathrm{PCR} /$ pyrosequencing, which permited earlier detection and an improvement of therapeutic management. 


\section{Discussions}

Low-abundance DNA mutations (e.g., EGFR T790M) in

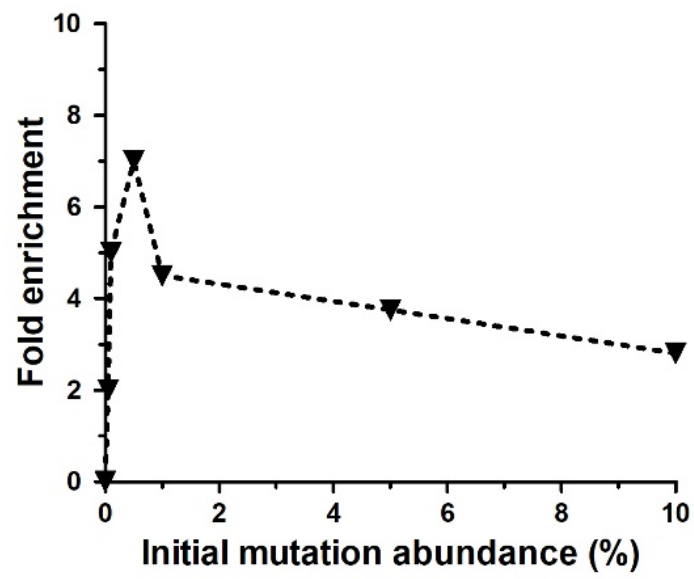

Fig. 3. Enrichment potential for COLD-PCR/pyrosequencing.

heterogeneous specimens may cause drug resistance and can be clinically significant biomarkers of disease progression. Therefore, it is crucial to design accurate and sensitive detection methods for such low-abundance mutations. Here, we have described COLD-PCR/pyroseuencing, a novel methodology that combined COLDPCR with downstream pyrosequencing for detecting the EGFR-encoded T790M mutant in serial dilutions of mutant DNA and in biological samples. As a result, the combination of COLD-PCR with pyrosequencing quantitatively detected as little as $0.1 \%$ mutant alleles with a 50-fold mutation enrichment, compared to conventional PCR/pyrosequencing. That was, COLD$\mathrm{PCR} /$ pyrosequencing exhibited higher levels of enrichment and sensitivity over conventional $\mathrm{PCR} /$ pyrosequencing. Our results were in agreement with a previous study ([20]). Furthermore, the detect limit of $0.1 \%$ in our work was slightly lower than that of the previously published work in which a COLD$\mathrm{PCR} /$ pyrosequencing assay has also been used for decte-

Table 1. Comparison of mutant-to-wild-type-ratio by COLD$\mathrm{PCR} /$ pyrosequencing and conventional PCR/pyrosequencing assays for the detection of EGFR T790M mutations. Samples with no T790M mutation identified by both conventional $\mathrm{PCR} /$ pyrosequencing and COLD-PCR/pyrosequencing were not listed.

\begin{tabular}{lcccc}
\hline No. & $\begin{array}{c}\text { Mutation } \\
\text { (aa) }\end{array}$ & $\begin{array}{c}\text { Ratio by } \\
\text { PCR }^{\mathrm{a}}(\%)\end{array}$ & $\begin{array}{c}\text { Ratio by } \\
\text { COLD- } \\
\text { PCR }^{\mathrm{b}}(\%)\end{array}$ & $\begin{array}{c}\text { Enhancement } \\
\text { efficiency } \\
\text { (fold) }\end{array}$ \\
\hline S1 & p.Thr790Met & 15 & 39 & 2.6 \\
S2 & p.Thr790Met & $\mathrm{NF}^{\mathrm{c}}$ & 10 & - \\
S6 & p.Thr790Met & 38 & 57 & 1.5 \\
S9 & p.Thr790Met & $\mathrm{NF}$ & 8 & - \\
S12 & p.Thr790Met & 16 & 30 & 1.9 \\
S15 & p.Thr790Met & 57 & 68 & 1.2 \\
S19 & p.Thr790Met & 25 & 35 & 1.4 \\
\hline a: Samples were & identified by conventional \\
PCR/pyrosequencing. b: Samples were identified by COLD- \\
PCR/pyrosequencing, c :not found.
\end{tabular}

\section{Acknowledgements}

The work was funded by the National Science

Foundation of China [61801071], the Basic Research

ting EGFR T790M, reducing the limit of detection to $0.125 \%$ and yielding a 40 -fold improved selectivity compared with standard PCR/pyrosequencing ([20]). COLD-PCR has also been applied to combine with HRM and TaqMan-based real-time PCR for the detection and identification of EGFR T790M mutation with a limit of detection of 0.01 and $0.8 \%$ respectively. $([16,17])$. Several studies have demonstrated that COLD-PCR exhibited high ability to identify much lower mutant fractions, improving the ability to detect minor allele variants in the early stages of disease progression $([14,21,22])$. Furthermore, COLD-PCR is cost effectiveness in comparison to other methods enabled to detect such small clones (eg, NGS) since it can be performed with conventional PCR reagents and laboratory equipment. These features allow it to become available to the normal routine processing without causing any additional costs.

Pyrosequencing is a relatively sensitive downstream technology, and the combination of pyrosequencing with COLD-PCR enables to detect as low as $0.1 \%$ of mutantDNA, making it widely be used as the downstream sequencing technology for mutation analysis ([23, 24]). In Fig. 2, pyrogram from $0 \%$ mutation-containing DNA sample also detected 1\% mutated alleles, it might result from the background signal. In addition, enrichments were represented as fold changes of the average mutation-to-wild-type ratios of the same dilution between COLD-PCR and conventional PCR amplifications. Here, the mutation-to-wild-type ratio was automatically shown upon the pyrogram by Pyromark Q96 MA software.

As for low-abundance mutation detection, a number of different factors might affect the sensitivity and the specificity, such as a low concentration of tumor cell, the presence of DNA fragment, poor quality of DNA, and non-sensitive downstream technologies. The combination of COLD-PCR with pyrosequencing enables to enrich the mutant content and to improve the detect limit of downstream technologies, providing a powerful tool for determinating low-abundance EGFR T790M mutations in clinical samples.

\section{Conclusion}

In conclusion, we have combined COLD-PCR with pyrosequencing for detecting EGFR T790M, and demonstrated that the use of COLD$\mathrm{PCR} /$ pyrosequencing increased the sensitivity for detecting EGFR T790M mutation by approximately 50fold with the limit of detection of $0.1 \%$ and increased the T790M mutation detected in clinical NSCLC tumor specimens by $10 \%(2 / 20)$. All in all, COLD$\mathrm{PCR} /$ pyrosequencing is high sensitivity, cost-effective and ease of use for identifying low-abundance EGFR T790M mutation in clinical specimens. 
and Frontier Exploration Project of Chongqing Science and Technology Commission [CSTC2018jcyjAX0246].

\section{References}

1. W. Wang, Z. Song, Y. Zhang, Cancer medicine 6, 154-162 (2017).

2. R. Rosell, T. G. Bivona, N. Karachaliou, Lancet 382, 720-731 (2013).

3. G. R. Oxnard, M. E. Arcila, C. S. Sima, G. J. Riely, J. Chmielecki, M. G. Kris, W. Pao, M. Ladanyi, V. A. Miller, Clin Cancer Res 17, 1616-1622 (2011).

4. Y. L. Wu, R. Z. Tong, Y. Zhang, B. B. Hu, K. Zheng, Z. Y. Ding, F. Peng, Y. L. Gong, Y. M. Liu, Y. Lu, Oncotargets Ther 10, 3307-3312 (2017).

5. L. Lettig, N. Sahnane, F. Pepe, R. Cerutti, C. Albeni, F. Franzi, G. Veronesi, F. Ogliari, A. Pastore, A. Tuzi, G. Pinotti, A. Bovio, C. Verusio, M. Giordano, G. Troncone, F. Sessa, U. Malapelle, D. Furlan, Transl Lung Cancer R 8, 584 (2019).

6. C. Martinez-Carretero, F. I. Pascual, A. Rus, I. Bernardo, Clin Chem Lab Med 55, 1970-1978 (2017).

7. M. Dono, G. De Luca, S. Lastraioli, G. Anselmi, M. G. Dal Bello, S. Coco, I. Vanni, F. Grossi, A. Vigani, C. Genova, M. Ferrarini, J. L. Ravetti, S. Zupo, Mol Med 25, 15 (2019).

8. M. G. Denis, A. Vallee, S. Theoleyre, Clin Chim Acta 444, 81-85 (2015).

9. H. D. Do, A. Dobrovic, Clin Chem 61, 64-71 (2015).

10. F. Mauger, A. How-Kit, J. Tost, Mol Diagn Ther 21, 269-283 (2017).

11. S. Ogino, T. Kawasaki, M. Brahmandam, L. Y. Yan, M. Cantor, C. Namgyal, M. Mino-Kenudson, G. Y. Lauwers, M. Loda, C. S. Fuchs, J Mol Diagn 7, 413421 (2005).

12. J. Tost, Expert Rev Mol Diagn 16, 265-268 (2016).

13. A. Ahmadian, B. Gharizadeh, A. C. Gustafsson, F. Sterky, P. Nyren, M. Uhlen, J. Lundeberg, Anal Biochem 280, 103-110 (2000).

14. J. Li, L. L. Wang, H. Mamon, M. H. Kulke, R. Berbeco, G. M. Makrigiorgos, Nat Med 14, 579-584 (2008).

15. 15 J. Li, G. M. Makrigiorgos, Biochem Soc T 37, 427-432 (2009).

16. J. Li, L. L. Wang, P. A. Janne, G. M. Makrigiorgos, Clin Chem 55, 748-756 (2009).

17. S. Hashida, J. Soh, S. Toyooka, T. Tanaka, M. Furukawa, K. Shien, H. Yamamoto, H. Asano, K. Tsukuda, K. Hagiwara, S. Miyoshi, Oncol Rep 32, 145-152 (2014).

18. D. Pu, R. F. Pan, W. N. Liu, P. F. Xiao, Electrophoresis 38, 876-885 (2017).

19. E. Castellanos-Rizaldos, P. F. Liu, C. A. Milbury, M. Guha, A. Brisci, L. Cremonesi, M. Ferrari, H. Mamon, G. M. Makrigiorgos, Clin Chem 58, 11301138 (2012).
20. F. D. Mairinger, C. Vollbrecht, A. Streubel, A. Roth, O. Landt, H. F. Walter, J. Kollmeier, T. Mairinger, Appl Immunohistochem Mol Morphol 22, 114-118 (2014).

21. C. A. Milbury, J. Li, P. F. Liu, G. M. Makrigiorgos, Expert Rev Mol Diagn 11, 159-169 (2011).

22. C. A. Milbury, J. Li, G. M. Makrigiorgos, Nucleic Acids Res 39, e2 (2011).

23. P. Pinzani, C. Santucci, I. Mancini, L. Simi, F. Salvianti, N. Pratesi, D. Massi, V. De Giorgi, M. Pazzagli, C. Orlando, Clin Chim Acta 412, 901-905 (2011).

24. W. Q. Jiang, W. B. Wang, F. F. Fu, X. D. Teng, H. H. Wang, H. Y. Wang, L. S. Teng, Mol Cell Biochem 366, 49-58 (2012). 